PROCEEDINGS OF THE

AMERICAN MATHEMATICAL SOCIETY

Volume 33, Number 1, May 1972

\title{
A SET-THEORETIC PROPOSITION IMPLYING THE METRIZABILITY OF NORMAL MOORE SPACES
}

\author{
FRANKLIN D. TALL ${ }^{1}$
}

\begin{abstract}
A set-theoretic proposition is shown to be equivalent to a topological statement implving that all first countable normal Hausdorff spaces are collectionwise normal, and hence that all normal Moore spaces are metrizable.
\end{abstract}

The question of whether every normal Moore space is metrizable has occupied a number of topologists. The purpose of this note is to give a purely set-theoretic formulation of a somewhat more general question, which may be of interest to a wider audience. Our methods and results extend those of Bing [2].

We first recall some definitions and make some new ones.

Definition 1. A development of a space $X$ is a sequence $\left\{G_{n}\right\}_{n \in \omega}$ of open covers of $X$, such that for each $p \in X$, and each open set $U$ containing $p$, there is an $n$ such that every element of $G_{n}$ which contains $p$ is contained in U. A space is developable if there is a development of it. A Moore space is a regular Hausdorff developable space.

Definition 2. A collection $\mathscr{Y}$ of subsets of a space $X$ is

(a) discrete if for each $p \in X$, there is an open set about $p$ intersecting at most one miember of $\mathscr{Y}$.

(b) normalized if for each $\mathscr{Z} \subset \mathscr{Y}$ there exist disjoint open sets $U, U^{\prime}$, such that $\bigcup\{Y: Y \in \mathscr{Z}\} \subset U, \bigcup\{Y: Y \in \mathscr{Y}-\mathscr{Z}\} \subset U^{\prime}$.

(c) separated if there exist mutually disjoint open sets $U_{Y}$, each $Y \in \mathcal{Y}$, $Y \subset U_{Y}$.

Definition 3. A space is

(a) normal if every discrete collection is normalized.

(b) collectionwise normal if every discrete collection is separated. (These last two are easily seen to be equivalent to the usual definitions.)

Presented to the Society October 27, 1970; received by the editors January 20, 1971.

AMS 1970 subject classifications. Primary 54D15, 54E30, 54E35, 02K99; Secondary 02K05, 02K25, 04A05, 04A10, 04A20, 04A30.

Key words and phrases. Moore space, metrizable, normal, collectionwise normal, first countable.

1 These results are part of the author's doctoral dissertation [3], which was supported by NSF grants GP-5913 and GP-8501. The preparation of this paper was assisted by grant A-7354 of the National Research Council of Canada.

American Mathematical Society 1972 
TheOREM 1 (BING [1]). A space is metrizable if and only if it is a collectionwise normal Moore space.

Definition 4. Let $\kappa$ and $\lambda, \lambda \leqq \kappa$, be infinite cardinals. Let ${ }^{*} \omega$ be the set of functions from $\kappa$ to $\omega$. Let $G \subset{ }^{\kappa} \omega$. Let $\mathscr{Y}=\left\{Y_{\gamma}\right\}_{; \in \lambda}$ be a collection of disjoint subsets of $\kappa$.

$\langle G, \mathscr{Y}\rangle$ is doubly superior if

$$
\begin{gathered}
\left(\forall f \in{ }^{\kappa}(\omega)\right)(\exists g \in G)\left(\exists \gamma_{0}, \gamma_{1} \in \lambda, \gamma_{0} \neq \gamma_{1}\right)\left(\exists \alpha_{0} \in Y_{\gamma_{0}}\right)\left(\exists \alpha_{1} \in Y_{\gamma_{1}}\right) \\
\left(g\left(\alpha_{0}\right)>f\left(\alpha_{0}\right), g\left(\alpha_{1}\right)>f\left(\alpha_{1}\right)\right) .
\end{gathered}
$$

Definition 5. Let $p \subset \lambda \times 2$ be a function, domain $p \subset \lambda$, range $p \subset 2$. $p$ splits $\langle G, \mathcal{Y}\rangle$ if $\left(\forall f \in^{\kappa}(1)\right)(\exists g \in G)\left(\exists \gamma_{0}, \quad \gamma_{1} \in \operatorname{domain} p, \quad p\left(\gamma_{0}\right) \neq p\left(\gamma_{1}\right)\right)$ $\left(\exists \alpha_{0} \in Y_{\gamma_{0}}\right)\left(\exists \alpha_{1} \in Y_{\gamma_{1}}\right)\left(g\left(\alpha_{0}\right)>f\left(\alpha_{0}\right), g\left(\alpha_{1}\right)>f\left(\alpha_{1}\right)\right)$.

$\langle G, \mathscr{Y}\rangle$ splits if there is a $p$ which splits $\langle G, \mathscr{Y}\rangle$.

THEOREM 2. Every normalized collection in every first countable $T_{1}$ space is separated if and only if for every $\kappa$ and $\lambda$, every doubly superior $\langle G, \mathscr{Y}\rangle$ splits.

COROLlaRY. If for every $\kappa$ and $\lambda$, every doubly superior $\langle G, \mathscr{Y}\rangle$ splits, then every normal Moore space is metrizable.

The corollary follows immediately from Theorerns 1 and 2, since Moore spaces are obviously first countable. To prove Theorem 2, first suppose $X$ is a first countable $T_{1}$ space and $\mathscr{Y}=\left\{Y_{\gamma}\right\}_{y \in \lambda}$ is a normalized collection in $X$ which is not separated. Let $\mathscr{Y} *=\{Y: Y \in Y Y\}$ be identified with $\kappa$, its cardinality. Since $M$ is normalized and $X$ is first countable, there is for each $\alpha \in Y_{y}$ a neighbourhood base $\{N(\alpha, n)\}_{n \in \omega^{\prime}}$ such that no $N(\alpha, n)$ intersects any $Y_{\gamma^{\prime}}, \gamma^{\prime} \neq \gamma$, and for each $n, N(\alpha, n) \supset N(\alpha, n+1)$. By $T_{1}$, there is for each $x \in X-y^{*}$, a $g_{x} \in^{\kappa}(1)$ defined by

$g_{x}(\alpha)=0$ if for no $n$ is $x \in N(\alpha, n)$,

$g_{x}(\alpha)=1$ plus the largest $n$ such that $x \in N(\alpha, n)$ otherwise.

Let $G=\left\{g_{x}\right\}_{x \in X^{\prime}-y}$. Claim $\left\langle G, y_{\rangle}\right.$is doubly superior but does not split.

Doubly superior. Let $f \in^{*}(1)$. Let $U_{y}=\bigcup\left\{N(\alpha, f(\alpha)): \alpha \in Y_{\gamma}\right\}$. Then for each $\gamma, U_{\gamma}$ is an open set containing $Y_{\gamma}$. Since $y$ is not separated, there exist $\gamma_{0} \neq \gamma_{1}$, such that $U_{\gamma_{0}} \cap U_{\ddot{\gamma}_{1}} \neq 0$. Let $x \in U_{i_{0}} \cap U_{\gamma_{1}}$. Then

$$
\left(\exists \alpha_{0} \in Y_{r_{0}}\right)\left(\exists \alpha_{1} \in Y_{r_{1}}\right)\left(x \in N\left(\alpha_{0}, f\left(\alpha_{0}\right)\right) \cap N\left(\alpha_{1}, f\left(\alpha_{1}\right)\right)\right) .
$$

But then $g_{x}\left(\alpha_{0}\right)>f\left(\alpha_{0}\right), g_{x}\left(\alpha_{1}\right)>f\left(\alpha_{1}\right)$.

Does not split. Suppose on the contrary that $p$ splits $\langle G, y\rangle$. Let $p_{0}=\{\gamma: p(\gamma)=0\}, p_{1}=\{\gamma: p(\gamma)=1\}$. Iy is normalized, so there exist disjoint

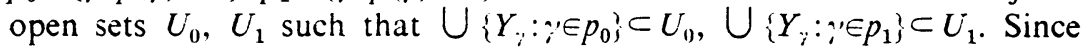
$\{N(\alpha, n)\}_{n \in \omega}$ is a neighbourhood base for $\alpha$, we may define $f \epsilon^{\kappa}(1)$ so that 
$N(\alpha, f(\alpha))=U_{0}$ or $U_{1}$. according to whether $\alpha \in Y_{\eta}, \gamma \in p_{0}$, or $\alpha \in Y_{\eta}, \gamma \in p_{1}$. We have assumed $p$ splits $G, Y$, hence

$$
\begin{gathered}
\left(\exists g_{x} \in G\right)\left(\exists \gamma_{\gamma_{0}} \in p_{0}\right)\left(\exists \gamma_{1} \in p_{1}\right)\left(\exists \alpha_{\alpha_{0}} \in Y_{\gamma_{0}}\right)\left(\exists \alpha_{1} \in Y_{\gamma_{1}}\right) \\
\left(g_{x}\left(\alpha_{0}\right)>f\left(\gamma_{0}\right), g_{x}\left(\alpha_{1}\right)>f\left(\alpha_{1}\right)\right) .
\end{gathered}
$$

But then $x \in N\left(\alpha_{0} . f\left(\alpha_{0}\right)\right) \cap N\left(\alpha_{1}, f\left(\alpha_{1}\right)\right) \subset U_{0} \cap U_{1}$, contradiction.

Conversely, suppose there is a doubly superior $\langle G, \mathscr{Y}\rangle$ which does not split. Let $\mathscr{Y}^{*}=\bigcup\{Y: Y \in \mathscr{Y}\}$. Let $X$ be the disjoint union of $G$ and $\mathscr{Y}^{*}$ with the following topology: each $\{g\}, g \in G$, is open; a neighbourhood base for $x \in \mathscr{y}^{*}$ is given by $\{N(\alpha, n)\}_{n \in \omega}$, where $N(\boldsymbol{x}, n)=\{\alpha\} \cup\{g \in G$ : $g(\alpha)>n\}$. It is then routine to verify that $X$ is a first countable $T_{1}$ space, and that $\mathscr{Y}$ is a normalized collection in $X$ which is not separated.

This completes the proof of Theorem 2 . The $T_{1}$ restriction can be removed by considering functions from $\kappa$ into $\omega+1$, rather than into $\omega$. In the general case, one has to consider functions from $\kappa$ which for each $\alpha$ take values in a directed set corresponding to a neighbourhood base for $\alpha$.

Bing's translation-although he approached the problem from a different perspective-amounted to considering the trivial partition $\left\{\{\alpha\}_{x \in \kappa}\right.$. In that case, we speak of $G$ (rather than $\left.G, Y\right\}$ ) being doubly superior or splitting. (Bing bears no responsibility for these terms.)

DEFINITION 6. A space is collectionwise Hausdorff if every discrete collection of points is separated.

A speciai case of Theorem 2 is then

THEOREM 3. Every first countable normal Hausdorff space is collectionwise Hausdorff if and only if for every $\kappa$, every doubly superior $G$ splits.

Definition 7. $G \subset^{\kappa^{\prime}()}$ is bounded if $(\forall g \in G)(\exists n \in(\cdot)(\forall \alpha \in \kappa)(g(\alpha) \leqq n)$. The proof of Theorem 2 now yields

THEOREM 4 (BING). Every normal Moore space is collectionwise Hausdorff if and only if for every $\kappa$, every doubly superior bounded $G$ splits.

The special case of whether there is a separable, normal, nonmetrizable Moore space admits a variety of felicitous set-theoretic translations. These are listed in [4], which summarizes the main results of [3]. We mention only one here:

THEOREM 5. Every separable normal Moore space is metrizable if and only if every countable $G \subset{ }^{\omega_{1}}(1)$ satisfying the following condition splits.

$$
\left(\forall \alpha \in\left(\omega_{1}\right)(\forall n \in(0)(\exists g \in G)(g(\alpha)>n) .\right.
$$

Aside from its topological import, the question of whether doubly superior collections split-especially $G$ 's rather than $G, \mathscr{Y}$ 's-seems to be 
a natural one from the point of view of a set-theorist. It is also natural to generalize the problem by replacing (1) by an arbitrary cardinai $\mu$. The one positive result is

THEOREM 6. Let $G \subset^{\star} \mu$. Let 3 be a collection of disjoint subsets of $\kappa$. If $\kappa \leqq \mu,\langle G, \mathcal{Y} \backslash$ doubly superior implies $\{G, y$, splits.

An easy way to prove this theorem is to translate into topology and then generalize the proof of the fact that countable normalized collections are separated. The point is that in the associated space, intersections of fewer than $\mu$ open sets are open, unless $\mu$ is singular. The singular case goes through using induction on previous cardinals.

When $\kappa>\mu$, the situation is unclear, which is to be expected, given the amount of time that has been devoted to the normal Moore space conjecture. A partial result is the following theorem which depends in varying degrees on work of Bing, R. M. Solovay, J. H. Silver, and the author.

THEOREM 7. The assertion that every doubly superior $G \subset^{\omega_{1}} \omega$ splits is consistent with, and independent of, the axioms of Zermelo-Fraenkel set theory (inciuding the Axiom of Choice).

Other partial results and the proof of Theorem 7 can be found-in translation-in [3].

\section{REFERENCES}

1. R. H. Bing, Metrization of ropological spaces, Canad. J. Math. 3 (1951), 175-186. MR 13, 264.

2. - A translation of the normal Moore space conjecture, Proc. Amer. Math. Soc. 16 (1965), 612-619. MR 31 \#6201.

3. F. D. Tall, Set-theoretic consistency resilts and topological theorems concerning the normal Moore space conjecture and related problems, Thesis, University of Wisconsin, Madison, Wis., 1969.

4. - New results on the normal Muore space problem, Proc. Conf. General Topology, Washirgton State University, Pullman, Wash., 1970, pp. 120-125.

Department of Mathematics, University of Toronto, Toronto, Ontario, Canada 